\title{
Feature tracking cardiac magnetic resonance imaging for the evaluation of myocardial strain in patients with dilated cardiomyopathy and in healthy controls
}

\author{
Kristin Breuninger ${ }^{1}$, Stephanie Lehrke², Philipp Matheis ${ }^{1}$, Yannick Sander ${ }^{1}$, Rebekka Kammerer ${ }^{1}$, Lukas Rust ${ }^{1}$, \\ Christian Galuschky ${ }^{3}$, Hugo A Katus', Grigorios Korosoglou', Sebastian Buss ${ }^{1 *}$
}

From 16th Annual SCMR Scientific Sessions

San Francisco, CA, USA. 31 January - 3 February 2013

\section{Background}

In clinical routine, quantification of myocardial strain using CMR tagging is currently the gold standard. Additional pulse sequences for the generation of tagged images and specialized software for the quantification of myocardial strain is necessary, so that alternative ways using conventional steady-state-free-precession (SSFP) sequence images would be preferable. This advantage may be ensured by feature tracking imaging algorithm, a novel method of two-dimensional deformation analysis.

To quantify myocardial deformation with two-dimensional feature tracking cardiac magnetic resonance (CMR) in patients with heart failure due to nonischemic cardiomyopathy and in healthy controls.

\section{Methods}

Eighty-eight patients with dilated cardiomyopathy and thirty healthy subjects were examined in a 1.5T CMRscanner. SSFP cine sequences of the four chamber view and mid-ventricular short axis view were analyzed using feature tracking imaging software (2D CPA MR@), TomTec Imaging Systems $\mathrm{GmbH}$ ). Generated parameters of the myocardial quantification were circumferential and longitudinal strain, respectively. Furthermore, patients were divided in subgroups classified by left-ventricular ejection-fraction $\mathrm{LV}-\mathrm{EF} \leq 35 \%$ and $\mathrm{EF}>35 \%$ and in patients with the presence or absence of late-gadolinium enhancement (LGE), respectively.

\section{Results}

In patients with dilated cardiomyopathy, close correlation were observed for the LV-EF with circumferential strain $\left(\mathrm{r}^{\wedge} 2=0.8, \mathrm{p}<0.0001\right)$ and with longitudinal strain $\left(\mathrm{r}^{\wedge} 2=0.5, \mathrm{p}<0.0001\right)$, respectively. Regression analysis showed also high correlation between NT-proBNP and circumferential strain $\left(\mathrm{r}^{\wedge} 2=0.47, \mathrm{p}<0.0001\right)$. Furthermore, significant differences of circumferential and longitudinal strain parameters were observed between healthy volunteers and patients with LV-EF $>35 \%$ and with $\mathrm{LV}-\mathrm{EF} \leq 35 \%(\mathrm{p}<0.0001)$. In addition, patients with LGE yielded significant lower circumferential and longitudinal strain values, compared to those without LGE $(\mathrm{p}<0.0001$ and $\mathrm{p}=0.02$, respectively).

\section{Conclusions}

Feature tracking imaging determines global myocardial function in patients with dilated cardiomyopathy and provides further insight into the underlying remodeling processes. Further investigation is necessary to analyze the impact of this new method on clinical outcome.

\section{Funding}

none

${ }^{1}$ University of Heidelberg, Heidelberg, Germany

Full list of author information is available at the end of the article 


\section{Author details}

${ }^{1}$ University of Heidelberg, Heidelberg, Germany. ${ }^{2}$ Diagnostic and

Interventional Radiology and Neuroradiology, Ev.- Lutherische

Diakonissenanstalt Flensburg, Flensburg, Germany. ${ }^{3}$ TomTec Imaging

Systems, Munich, Germany.

Published: 30 January 2013

doi:10.1186/1532-429X-15-S1-P167

Cite this article as: Breuninger et al.: Feature tracking cardiac magnetic resonance imaging for the evaluation of myocardial strain in patients with dilated cardiomyopathy and in healthy controls. Journal of Cardiovascular Magnetic Resonance 2013 15(Suppl 1):P167.

Submit your next manuscript to BioMed Central and take full advantage of:

- Convenient online submission

- Thorough peer review

- No space constraints or color figure charges

- Immediate publication on acceptance

- Inclusion in PubMed, CAS, Scopus and Google Scholar

- Research which is freely available for redistribution

Submit your manuscript at www.biomedcentral.com/submit
C Biomed Central 